\title{
About uniformly Lindelöf spaces
}

\author{
Bekbolot E. Kanetov, Ulukbek A. Saktanov and \\ Meerim O. Zhanakunova
}

Communicated by Ljubisa Kocinac

\begin{abstract}
The idea of Lindelöfness is one of the most important in General Topology. There have been some variants in defining uniformly Lindelöfness of uniform spaces. For example, uniform $A$-Lindelöfness in the sense of L.V. Aparina [1], uniform $B$-Lindelöfness in the sense of A.A. Borubaev [2], uniform $I$-Lindelöfness in the sense of D.R. Isbell [5].

In this paper we propose a new approach to the definition of a uniform analogue of Lindelöfness. We introduce and study uniformly Lindelöf spaces.
\end{abstract}

Keywords. Finitely-additive open covering, locally finite uniform covering, $\aleph_{0}$-boundedness, uniformly perfect mappings.

2020 Mathematics Subject Classification. 54E15, 54D20.

\section{Introduction}

Throughout this work all uniform spaces are defined in terms of coverings, are assumed to be Hausdorff and mappings are uniformly continuous [4].

For coverings $\alpha$ and $\beta$ of the set $X$, the symbol $\alpha \succ \beta$ means that the covering $\alpha$ is a refinement of the covering $\beta$, i.e. for any $A \in \alpha$ there exists $B \in \beta$ such that $A \subset B$ and, for coverings $\alpha$ and $\beta$ of a set $X$, we have: $\alpha \wedge \beta=\{A \cap B: A \in$ $\alpha, B \in \beta\}$. The covering $\alpha$ is finitely-additive if $\alpha^{L}=\alpha$, where $\alpha^{L}=\left\{\bigcup \alpha_{0}\right.$ : $\alpha_{0} \subset \alpha$ is finite $\} . \alpha(x)=\bigcup S t(\alpha, x), \operatorname{St}(\alpha, x)=\{A \in \alpha: A \ni x\}, x \in X$, $\alpha(M)=\bigcup \operatorname{St}(\alpha, M), \operatorname{St}(\alpha, M)=\{A \in \alpha: A \bigcap M \neq \emptyset\}, M \subset X$.

For a uniform space $(X, U)$ by $\tau_{U}$ we understand the topology generated by the uniformity $U$; for the Tychonoff space $X$ by $U_{X}$ we understand the universal uniformity.

A uniform space $(X, U)$ is called:

(1) uniformly $A$-Lindelöf, if for each open covering $\alpha$ exist a countable uniform covering $\beta=\left\{B_{n}: n \in N\right\}$ and $\gamma \in U$ such that $\beta \succ \alpha^{<}$and $\gamma\left(\bar{B}_{n}\right) \subset B_{n+1}$ for any $n \in N[1]$;

(2) uniformly $B$-Lindelöf, if it is both uniformly $B$-paracompact and $\aleph_{0}$-bounded [2]; 
(3) uniformly $I$ - Lindelöf, if every uniform covering has a locally finite uniform refinement [5];

(4) uniformly $A$-paracompact, if every open covering has a locally finite uniform refinement [1];

(5) strongly uniformly $A$-paracompact, if every open covering has a star finite uniform refinement [4];

(6) uniformly $B$-paracompact, if for each finitely-additive open covering $\lambda$ of $(X, U)$ there exists a sequence $\left\{\alpha_{i}: i \in N\right\} \subset U$ of uniform coverings such that the following condition is fulfilled: for each point $x \in X$ there exist number $i \in N$ and $L \in \lambda$ such that $\alpha_{i}(x) \subset L(\mathrm{U})$ [2];

(7) $\aleph_{0}$-bounded if the uniformity $U$ has a base consisting of countable coverings [2];

(8) strongly uniformly locally compact if the uniformity of $U$ contains a locally finite uniform covering such that the closure of each its element is compact [1];

(9) uniformly locally Lindelöf if the uniformity of $U$ contains a uniform covering whose closure of each element is Lindelöf [3];

For covering properties of uniform spaces close to $\aleph_{0}$-boundedness and Lindelöfness see [7].

A uniformly continuous mapping $f:(X, U) \rightarrow(Y, V)$ of uniform space $(X, U)$ onto a uniform space $(Y, V)$ is called:

(1) precompact, if for each $\alpha \in U$ there exist a uniform covering $\beta \in V$ and finite uniform covering $\gamma \in U$, such that $f^{-1} \beta \wedge \gamma \succ \alpha$ [2];

(2) uniformly perfect, if it is both precompact and perfect [2];

(3) uniformly open, if $f$ maps each open uniform covering $\alpha \in U$ to an open uniform covering $f \alpha \in V$ [2].

\section{Uniformly Lindelöf spaces}

Let $(X, U)$ be a uniform space.

Definition 2.1. A uniform space $(X, U)$ is said to be uniformly Lindelöf, if it is both uniformly $A$-paracompact and $\aleph_{0}$-bounded.

Proposition 2.2. If $(X, U)$ is a uniformly Lindelöf space, then its topological space $\left(X, \tau_{U}\right)$ is Lindelöf. Conversely, if $(X, \tau)$ is Lindelöf topological space, then the uniform space $\left(X, U_{X}\right)$ is uniformly Lindelöf.

Proof. Let $\alpha$ be an arbitrary finitely-additive open covering of $\left(X, \tau_{U}\right)$. Then for covering $\alpha$ there exists a locally finite uniform covering $\beta$ such that $\beta \succ \alpha$. Since the space $(X, U)$ is $\aleph_{0}$-bounded the covering $\beta$ contains a countable uniform 
covering $\beta_{0}$. Then for any $i \in N$ we have $B_{i} \subset \bigcup_{j=1}^{k} A_{j}$. The system $\left\{B_{i} \cap A_{j}\right\}$, $i=1,2, \ldots, n, j=1,2, \ldots, k$ forms a countable open covering which is refinement of $\alpha$. Consequently, the space $\left(X, \tau_{U}\right)$ is Lindelöf.

Conversely, if $(X, \tau)$ is Lindelöf, then the system of all open coverings forms a base of universal uniformity $U_{X}$ of the space $(X, \tau)$. It follows from this that $\left(X, U_{X}\right)$ is uniformly Lindelöf.

Proposition 2.3. Any compact space is uniformly Lindelöf.

Proof. Any compact uniform space is precompact and uniformly $A$-paracompact. Consequently, the uniform space $(X, U)$ is uniformly Lindelöf.

Any uniformly Lindelöf space is uniformly $A$-paracompact.

Proposition 2.4. Any closed subspace of a uniformly Lindelöf space is uniformly Lindelöf.

Proof. Let $\left(M, U_{M}\right)$ be a subspace of $(X, U)$. Note that space $\left(M, U_{M}\right)$ is $\aleph_{0^{-}}$ bounded. Let $\alpha_{M}$ be an arbitrary finitely-additive open covering of the subspace $\left(M, U_{M}\right)$. Then there exits a finitely-additive open family $\alpha$ of $(X, U)$, such that $\alpha \wedge\{M\}=\alpha_{M}$. Assume $\beta=\{\alpha, X \backslash M\}$. Obviously the covering $\beta$ is a finitely-additive open covering of $(X, U)$. Then there exists a locally finite uniform covering $\lambda \in U$ such that $\lambda \succ \beta$. Evidently, $\lambda_{M} \in U_{M}$. Hence $\lambda_{M} \succ \alpha_{M}$. It is easy to check that $\lambda_{M}$ is a locally finite uniform covering of $\left(M, U_{M}\right)$. Thus, $\left(M, U_{M}\right)$ is uniformly Lindelöf .

Corollary 2.5. Any uniformly Lindelöf space is uniformly locally Lindelöf.

Corollary 2.6. Any strongly uniformly locally compact and $\aleph_{0}$-bounded space is uniformly Lindelöf.

Corollary 2.7. The real space $R$ with natural uniformity is uniformly Lindelöf.

Proof. It is clear that $R$ is $\aleph_{0}$-bounded. Let $\alpha$ be a finitely-additive open covering of $R$. Put $\beta=\{(n-1, n+1): n=0, \pm 1, \pm 2, \ldots\}$. Then $\beta$ is a locally finite uniform covering of $R$. Since $[n-1, n+1]$ is compact, there exists a finite family $\left\{A_{1}, A_{2}, \ldots, A_{n}\right\}, A_{i} \in \alpha, i=1,2, \ldots, n$ such that $(n-1, n+1) \subset[n-1, n+1] \subset$ $\bigcup_{i=1}^{n} A_{i}$. Therefore, $\beta \succ \alpha^{<}$. Hence, $R$ is uniformly Lindelöf.

Proposition 2.8. Any uniformly Lindelöf space is uniformly B-Lindelöf and any uniformly B-Lindelöf space is uniformly I-Lindelöf. 
Proof. Let $(X, U)$ be a uniformly Lindelöf space. As it is known, every uniformly $A$-paracompact space is uniformly $B$-paracompact [4]. Hence, the uniform space $(X, U)$ is uniformly $B$-Lindelöf.

Let $(X, U)$ be a uniformly $B$-Lindelöf space. Then by the definition of uniformly $B$-Lindelöfness [2] we have $(X, U)$ is a uniformly $I$-Lindelöf.

Corollary 2.9. Any uniformly Lindelöf space is uniformly I-Lindelöf.

Theorem 2.10. Any uniformly Lindelöf space is strongly uniformly A-paracompact.

Proof. Let $(X, U)$ be a uniformly Lindelöf space. Then from the facts that the space $(X, U)$ is strongly uniformly $A$-paracompact if and only if it is uniformly $A$-paracompact and $\left(X, \tau_{U}\right)$ is strongly paracompact, one concludes that $\mathrm{b}(X, U)$ is strongly uniformly $A$-paracompact.

Proposition 2.11. Any uniformly Lindelöf space is complete.

Proof. Let $(X, U)$ be a uniformly Lindelöf space. The completeness of $(X, U)$ follows from the fact that every strongly uniformly $A$-paracompact space is complete [4].

Noncomplete separable uniform space need not be uniformly Lindelöf. For example, the space $(0,1)$ is uniformly $B$-Lindelöf, but not uniformly Lindelöf.

Lemma 2.12. Let $f:(X, U) \rightarrow(Y, V)$ be a precompact mapping of a uniform space $(X, U)$ to a uniform space $(Y, V)$. If a space $(Y, V)$ is $\aleph_{0}$-bounded, then $(X, U)$ is also $\aleph_{0}$-bounded.

Proof. Let $f:(X, U) \rightarrow(Y, V)$ be a precompact mapping of a uniform space $(X, U)$ to a uniform space $(Y, V)$ and $\alpha \in U$ be an arbitrary uniform covering of $X$. Then by virtue of the precompactness of $f$ there exist such a countable covering $\beta \in V$ and a finite covering $\gamma \in U$ that $f^{-1} \beta \wedge \gamma \succ f^{-1} \alpha$. But the covering $f^{-1} \beta \wedge \gamma$ is countable. Therefore the space $(X, U)$ is $\aleph_{0}$-bounded.

Lemma 2.13. Let $f:(X, U) \rightarrow(Y, V)$ be a uniformly continuous mapping of a uniform space $(X, U)$ and $(Y, V)$. If $\beta$ is a locally finite uniform covering of the space $(Y, V)$, then $f^{-1} \beta$ is a locally finite uniform covering of the space $(X, U)$.

Proof. By the conditions of the lemma for each point $x \in X$ there exists a neighborhood $O_{x}$ such that $O_{x}$ meets at most a finite number of elements of the covering $\beta$, i.e. exist elements $B_{i} \in \beta, i=1,2, \ldots, n$, such $O_{x} \subset \bigcup_{i=1}^{n} B_{i}$. As the mapping 
$f$ is uniformly continuous, the covering $f^{-1} \beta$ is uniform, i.e. $f^{-1} \beta \in U$. Hence $f^{-1} O_{x} \subset \bigcup_{i=1}^{n} f^{-1} B_{i}, f^{-1} B_{i} \in f^{-1} \beta$. So, $f^{-1} \beta$ is a locally finite uniform covering of $(X, U)$.

Lemma 2.14. Let $f:(X, U) \rightarrow(Y, V)$ be a uniformly continuous mapping of a uniform space $(X, U)$ to uniform space $(Y, V)$. If a space $(X, U)$ is $\aleph_{0}$-bounded, then $(Y, V)$ is also $\aleph_{0}$-bounded.

Proof. Let $\beta \in V$ be an arbitrary uniform covering. Then $f^{-1} \beta \in U$. According to Proposition 1.1.6. [2, p. 40] the covering $f^{-1} \beta$ has a countable subcovering $f^{-1} \beta_{0}$. Then $\beta_{0}$ is a countable subcovering of $\beta$. Therefore the space $(Y, V)$ is $\aleph_{0}$-bounded.

Theorem 2.15. Let $f:(X, U) \rightarrow(Y, V)$ be a uniformly perfect mapping of a uniform space $(X, U)$ onto a uniformly Lindelöf space $(Y, V)$. Then $(X, U)$ is uniformly Lindelöf.

Proof. According to Lemma 12 the space $(X, U)$ is $\aleph_{0}$-bounded. Let the uniform space $(Y, V)$ be uniformly $A$-paracompact and $\alpha$ be an arbitrary finitely-additive open covering of $(X, U)$. Then $\left\{f^{-1} y: y \in Y\right\} \succ \alpha$. Since the mapping $f$ is closed, it follows that $\lambda=\left\{f^{\#} A: A \in \alpha\right\}$ is a finitely-additive open covering of $(Y, V), f^{\#} A=Y \backslash f(X \backslash A)$. By virtue of the Lindelöfness of the space $(Y, V)$ there exist a locally finite covering $\beta \in V$ such that $\beta \succ \lambda$. Then $f^{-1} \beta \succ \lambda$ and according to Lemma 13 the covering $f^{-1} \beta$ is locally finite. So, $(X, U)$ is uniformly Lindelöf.

Proposition 2.16. Let $f:(X, U) \rightarrow(Y, V)$ be a uniformly open mapping of a uniform space $(X, U)$ onto a uniform space $(Y, V)$. If $(X, U)$ is a uniformly Lindelöf space, then $(Y, V)$ is also uniformly Lindelöf.

Proof. Let $f$ be a uniformly open mapping of a uniform space $(X, U)$ onto a uniform space $(Y, V)$ and $\alpha$ be an arbitrary finitely-additive open covering of $Y$. Then $f^{-1} \alpha$ is a finitely-additive open covering of the space $(X, U)$ and due to the uniform $A$-paracompactness where is a locally finite uniformly open covering $\beta \in U$ of $f^{-1} \alpha$. Since $f$ is open, $f(\beta) \succ \alpha$. Then $(Y, V)$ is uniformly $A$ paracompact. Note that $(Y, V)$ is $\aleph_{0}$-bounded. So, the space $(Y, V)$ is uniformly Lindelöf. 


\section{Bibliography}

[1] L.V. Aparina, Uniform Lindelöf spaces, Trudy Mosk. Math. Obshestva 57 (1996), 3-15 (in Russian).

[2] A.A. Borubaev, Uniform Spaces and Uniformly Continuous Mappings, Ilim, Frunze, 1990 (in Russian).

[3] D. Buhagiar and B. Pasynkov, On uniform paracompactness, Czech Math. J. 46(121) (1996), 577-586.

[4] R. Engelking, General Topology, 2nd edition, vol. 6 of Sigma Series in Pure Mathematics, Heldermann Verlag, Berlin, 1989.

[5] J.R. Isbell, Uniform Spaces, American Mathematical Society, Providence, 1964.

[6] B.E. Kanetov, Some Classes of Uniform Spaces and Uniformly Continuous Mappings, Bishkek, 2013 (in Russian).

[7] L.D.R. Kočinac, Selection principles in uniform spaces, Note Mat. 22(2) (2003/2004), 127-139.

Received February 7, 2021; revised July 17, 2021; accepted August 31, 2021.

\section{Author information}

Bekbolot E. Kanetov, Jusup Balasagyn Kyrgyz National University, Bishkek,

Kyrgyzstan.

E-mail: bekbolot_kanetov@mail.ru

Ulukbek A. Saktanov, Osh State University, Osh, Kyrgyzstan.

E-mail: uca73@mail.ru

Meerim O. Zhanakunova, Kuseyin Karasaev Bishkek Humanities University, Bishkek, Kyrgyzstan.

E-mail: aelin_jasmin@mail.ru 